\title{
Características clínicas y sociodemográficas de una población adulta con tumores neuroendocrinos
}

\section{Clinical and sociodemographic characteristics of an adult population with neuroendocrine tumors}

Correspondencia

Deiner Yivelson Muñoz-Delgado deineryiv@gmail.com

Recibido: 01/03/2021

Arbitrado por pares

Aprobado: 22/09/2021

Citar como: Martínez Montalvo CM, Muñoz-Delgado DY, GómezLedesma YR, Pérez-Hettinga MA, Vargas-Calderón MD, Ordoñez-Muñoz SG, Pinzón-Tovar A, ConradoJiménez H. Características clínicas y sociodemográficas de una población adulta con tumores neuroendocrinos. Acta Med Peru. 2021;38(3):18692. doi: https://doi.org/10.35663/ amp.2021.383.2058
Carlos Mauricio Martínez Montalvo ${ }^{1 a}$, Deiner Yivelson Muñoz Delgado ${ }^{2 b}$, Yeison Rodolfo Gómez Ledesma ${ }^{3 c}$, Manuel Antonio Pérez Hettinga ${ }^{1 a}$, María Daniela Vargas Calderon ${ }^{2 b}$, Silvia Gabriela Ordoñez Muñoz ${ }^{2 b}$, Alejandro Pinzón Tovar ${ }^{4 d}$, Héctor Conrado Jiménez ${ }^{5 e}$.

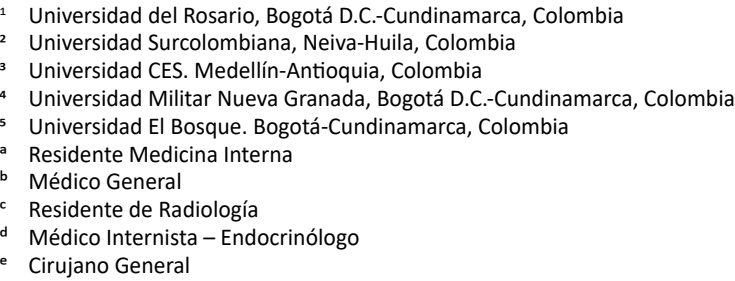

\section{RESUMEN}

Introducción: Los tumores neuroendocrinos representan un grupo de neoplasias de baja incidencia que derivan de células neuroendocrinas distribuidas en todo el cuerpo en especial sistema respiratorio y gastrointestinal. Objetivo: Determinar las características clínicas y sociodemográficas de una población adulta con padecimiento de tumores neuroendocrinos. Materiales y métodos: Estudio descriptivo transversal, se evaluaron 91 historias clínicas con diagnóstico de tumores neuroendocrinos confirmados por patología entre los años 2013 a 2020. Análisis realizado en Microsoft Excel 2013 y Epilnfo 7.2. Resultados: La media de edad fue 60 años, con predominio en hombres (57\%). Los principales antecedentes fueron el tabaquismo (35\%), hipertensión arterial (22\%) y EPOC (9\%). Los principales síntomas fueron el dolor abdominal (43\%), pérdida de peso (31\%) y tos (26\%). Según el origen, fueron más frecuentes los de intestino anterior (75\%), predominando los de tracto respiratorio (39,5\%). En el 21,9\%, el origen fue desconocido. Teniendo en cuenta la clasificación 2019 de la OMS, predominaron los carcinomas neuroendocrinos (56\%), de los cuales el más frecuente fue el carcinoma de células pequeñas. Entre los bien diferenciados $(44 \%)$, fueron más frecuentes los de bajo grado (58\%) seguido grado intermedio (24\%) y bajo grado (17\%). Las metástasis se registraron en $37 \%$ de los casos con afectación principalmente hepática (49\%), ganglios (21\%) y sistema nervioso central (9\%). La muerte se presentó en el $24 \%$ de los casos. Conclusiones: Los resultados del presente estudio concuerdan con lo reportado a nivel mundial, resaltando el predominio de los tumores de origen pulmonar, como también clínica semejante según los órganos afectados.

Palabras claves: Carcinoma Neuroendocrino, tumores neuroendocrinos (Fuente: DeCS BIREME). 


\begin{abstract}
Introduction: Neuroendocrine tumors represent a group of low-incidence neoplasms derived from neuroendocrine cells distributed throughout the body, especially the respiratory and gastrointestinal systems. Objective: To determine the clinical and sociodemographic characteristics of an adult population with neuroendocrine tumors. Materials and methods: In a descriptive cross-sectional study, 91 medical records with a diagnosis of neuroendocrine tumors confirmed by pathology were evaluated between the years 2013 and 2020. Analysis carried out in Microsoft Excel 2013 and Epilnfo 7.2. Results: The mean age was 60 years, with a predominance in men (57\%). The main antecedents were smoking (35\%), arterial hypertension (22\%), and COPD (9\%). The main symptoms were abdominal pain (43\%), weight loss (31\%), and cough (26\%). According to the origin, those of the foregut were more frequent (75\%), predominantly those of the respiratory tract (39.5\%). In $21.9 \%$, the origin was unknown. Taking into account 2019 WHO classification, neuroendocrine carcinomas predominated (56\%), of which the most frequent was small cell carcinoma. Among the well-differentiated (44\%), low-grade (58\%) followed by intermediate grade (24\%) and low-grade (17\%). Metastases were registered in $37 \%$ of the cases with mainly liver involvement (49\%), lymph nodes (21\%), and central nervous system (9\%). Death occurred in $24 \%$ of cases. Conclusions: The results of the present study coincide with those reported worldwide, highlighting the predominance of tumors of pulmonary origin, as well as similar clinical symptoms according to the affected organs
\end{abstract}

Keywords: Neuroendocrine Carcinoma, Neuroendocrine tumors (Source: MeSH)

\section{INTRODUCCIÓN}

Los tumores neuroendocrinos (NET) son un grupo de tumores originados de las células del sistema neuroendocrino difuso de todo el cuerpo; su comportamiento puede ser benigno o maligno, y su distribución es especialmente en tejidos como pulmón, intestino delgado, recto, páncreas, estómago, apéndice cecal, colon y timo ${ }^{[1]}$. Estos tumores son considerados raros con una incidencia de 6,98 casos por cada 100.000 personas, que se ha incrementado en las últimas décadas ${ }^{[2]}$. Los NET representan el $0,49 \%$ de todas las neoplasias en general ${ }^{[3]}$, sus manifestaciones clínicas dependen de la capacidad funcional del tumor, que a su vez es debida a la secreción de hormonas y aminas, siendo uno de sus síndromes más comunes el síndrome carcinoide ${ }^{[4]}$.

Los signos y síntomas de los tumores neuroendocrinos varían dependiendo del órgano afectado, sin embargo, muchos de estos son comunes entre ellos. Los principales signos y síntomas son: El dolor (79-100\%), diarrea (32-84\%), deshidratación (83\%), hipopotasemia (80-100\%), pérdida de peso (66-96\%), diabetes mellitus (63-90\%), cardiopatías (11-41\%) y masa abdominal $(10-30 \%)^{[5]}$

Estos tumores se diferencian por propiedades de tinción histológica, utilizando técnicas de inmunohistoquímica de cromograninas ( $A, B$ y $C$ ) y sinaptofisina. Sin embargo, el marcador que más se usa es la cromogranina $A$. Para establecer un diagnóstico específico es necesario la presencia de signos clínicos de la enfermedad asociado a los resultados de estudios de inmunohistoquímica ${ }^{[6]}$. La OMS en su última actualización

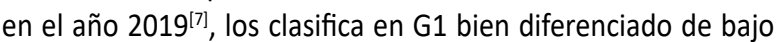
grado (Ki67 <3\%), G2 bien diferenciado de grado intermedio (Ki67 $3-20 \%$ ) y $G 3$ bien diferenciado de alto grado (Ki67> 20\%), según el índice de proliferación Ki67. Los G3 se clasifican además en dos categorías: bien diferenciados de alto grado (GEP-NET) o poco diferenciados de alto grado (carcinomas neuroendocrinos GEP, GEP-NEC), esta nueva clasificación es debido a mayor incidencia de origen pancreático en tumores G3 bien diferenciados los cuales muestran menor tasa de proliferación Ki67, mayor expresión de receptores de somatostatina y por tal su manejo es más conservador con mejores tasa de supervivencia, a diferencia de tumores poco diferenciados quienes son susceptibles de lesiones más extensas, manejos quirúrgicos más radicales, mayor de tasa de metástasis y mayor uso de terapia neoadyuvante, lo anterior dando un peor pronóstico ${ }^{[8]}$.

El objetivo del presente estudio fue determinar las características clínicas y sociodemográficas de pacientes con diagnósticos de tumores neuroendocrinos atendidos en un hospital de tercer nivel de Colombia, aportando datos del comportamiento de esta enfermedad en nuestra población.

\section{MATERIALES Y MÉTODOS}

Se realizó un estudio observacional descriptivo de corte transversal. Evaluamos 91 historias clínicas desde el 1 de enero de 2013 al 31 de enero de 2020, de pacientes mayores de 15 años con diagnósticos de tumores neuroendocrinos en el Hospital Universitario Hernando Moncaleano Perdomo (HUHMP), Neiva, Huila, Colombia. Se excluyeron historias sin información completa, con diagnostico extrainstitucional de tumores neuroendocrinos y pacientes menores de 15 años. Los datos extraídos de reportes finales de histopatología se correlacionaron con la base de datos de diagnóstico final de tumor neuroendocrino y posteriormente se realizó la revisión de historias clínicas.

Se registraron datos de características demográficas, presentación clínica y paraclínica, localización tumoral, grado de diferenciación 
a acuerdo a la última clasificación de la OMS del año 2019 (G1 (Ki67 <3\%), G2 (Ki67 3-20\%) y G3 (Ki67> 20\%)), localización según su origen embriológico e inmunohistoquímica, presencia de metástasis y mortalidad.

Los datos fueron registrados, procesados y analizados en Microsoft Excel 2013 y Epilnfo 7.0. Para las variables cuantitativas se analizaron mediante medidas de tendencia central y de dispersión, las cualitativas mediante frecuencias relativas, razón y proporción. El estudio fue formulado en concordancia con la reglamentación ética vigente mundial (declaración de Helsinki / Reporte Belmont / Pautas CIOMS) y la normativa (resolución 8430 de 1993) del ministerio de Salud de Colombia. Se obtuvo aprobación del Comité de Ética e investigación del Hospital Universitario Hernando Moncaleano Perdomo de Neiva - Huila Colombia (Acta aprobatoria 006-001 del 30 de julio del 2019). Lo anterior conlleva a que en toda la recolección de la información prevalezca el respeto a la dignidad de los sujetos en estudio, la protección de sus derechos y la confidencialidad tanto de los datos obtenidos como también de los pacientes estudiados salvaguardando el principio de confidencialidad de los pacientes.

\section{RESULTADOS}

De los 91 pacientes, 57\% fueron hombres, edad superior a 61 años $(62,2 \%)$, régimen social subsidiado y procedentes del área urbana (67\%) (Ver Tabla 1). Entre los antecedentes toxicológicos, el tabaquismo fue el más frecuente $(23,7 \%$ ) y entre los patológicos, la hipertensión arterial (22\%) seguida de EPOC $(9,9 \%)$ y diabetes mellitus (4,3\%) (ver Tabla 2$)$.

\section{Caracterización de los tumores neuroendocrinos.}

El estudio de inmunohistoquímica reportó con mayor frecuencia el marcador sinaptofisina (65.6\%), seguido por la cromogranina (57\%) y el CD56 (31\%) (Ver Tabla 2). En la clasificación histopatológica predominaron los carcinomas neuroendocrinos (56\%), de los cuales fue más frecuente el carcinoma de células pequeñas $(70,5 \%)$. De los tumores neuroendocrinos fue más frecuente el de bajo grado (58\%), seguido del grado intermedio o $\mathrm{G} 2$ (24\%).

Teniendo en cuenta la clasificación según su origen embriológico, se registró con mayor frecuencia tumores del intestino anterior como los tumores de origen pulmonar, bronquial y traqueal $(39,5 \%)$, seguidos de estómago (10,5\%). En intestino medio predominaron los tumores ubicados en apéndice $(6,5 \%)$ seguido por los de colon (4,4\%); Por último, en intestino posterior se encontró afectación por cáncer de recto en el 1\%. En un $21,9 \%$ su origen primario fue desconocido, diagnosticado por manifestaciones secundarias a las metástasis (Ver Figura 1).

Las metástasis se reportaron en un $37 \%$ de los pacientes, afectando diversos órganos, principalmente hepático (49\%), seguido por metástasis a ganglios (21\%) y en tercer lugar a sistema nerviosos central (9\%), Otros órganos afectados fueron el bazo,
Tabla 1. Características sociodemográficas.

\begin{tabular}{|c|c|}
\hline Característica & n (\%) \\
\hline Edad (años) & 60 (DE:18.2) * \\
\hline $15-29$ & $10(11.1)$ \\
\hline $30-60$ & $25(27.7)$ \\
\hline$>61$ & $56(62.2)$ \\
\hline \multicolumn{2}{|l|}{ Género } \\
\hline Masculino & $52(57.1)$ \\
\hline Femenino & $39(42.9)$ \\
\hline \multicolumn{2}{|l|}{ Estado civil } \\
\hline Soltero & $37(40.6)$ \\
\hline Casado & $30(32.9)$ \\
\hline Unión libre & $13(14.2)$ \\
\hline Viudez & $5(5.4)$ \\
\hline Separado & $6(6.6)$ \\
\hline \multicolumn{2}{|l|}{ Régimen social } \\
\hline Subsidiado & $71(78)$ \\
\hline Contributivo & $12(13.1)$ \\
\hline Vinculados & $3(3.2)$ \\
\hline Especial/particular & $5(5.4)$ \\
\hline \multicolumn{2}{|l|}{ Área de procedencia } \\
\hline Urbana & $61(67.0)$ \\
\hline Rural & $30(32.9)$ \\
\hline
\end{tabular}

* Media y la desviación estándar.

peritoneo, médula ósea y ovarios (Ver Figura 2). Estas metástasis fueron principalmente de los carcinomas neuroendocrinos $(61,5 \%)$, de las cuales predominaron los carcinomas de células pequeñas (62,5\%). Desde el inicio el estudio hasta la fecha actual se ha presentado la muerte de $24 \%$ de los pacientes.

\section{DISCUSIÓN}

Los TNE son entidades consideradas raras, pero en crecimiento en las últimas tres décadas, siendo en el segundo tumor más frecuente en el tracto gastrointestinal, con una incidencia mundial aproximada de 2,5 a 5,25 casos por 100.000 habitantes. Hay una mayor tasa de detección temprana de tumores localizados y mejor tasa de seguimiento en identificar posible metástasis. Su ubicación es variable, puede ser funcional según su producción hormonal y su clasificación histopatológica tiene en cuenta el índice proliferación y marcadores como Cromogranina, Sinaptofisina, entre otros. ${ }^{[9]}$

En nuestro estudio, los tumores fueron más frecuentes en hombres, similar a otros estudios, sin una marcada diferencia*. La mediana de edad fue de 60 años, comparable con otras series 
Tabla 2. Características clínicas.

\begin{tabular}{|c|c|}
\hline Característica & $n(\%)$ \\
\hline Índice de masa corporal & $22.3(4.5)^{*}$ \\
\hline Bajo peso & $15(16.4)$ \\
\hline Normal & $55(60.4)$ \\
\hline Sobrepeso y obesidad & $21(23)$ \\
\hline \multicolumn{2}{|l|}{ Antecedentes } \\
\hline Tabaquismo & $29(23.7)$ \\
\hline Alcoholismo & $3(3.3)$ \\
\hline Hipertensión arterial & $20(22)$ \\
\hline Enfermedad pulmonar obstructiva crónica & $9(9.9)$ \\
\hline Diabetes mellitus & $4(4.3)$ \\
\hline Insuficiencia cardiaca & $4(4.3)$ \\
\hline Infarto agudo de miocardio & $2(2.1)$ \\
\hline \multicolumn{2}{|l|}{ Signos y síntomas más frecuentes } \\
\hline Dolor abdominal & $39(43.3)$ \\
\hline Pérdida de peso & $28(31.1)$ \\
\hline Masa abdominal & $14(15.6)$ \\
\hline Tos & $23(26.4)$ \\
\hline Diarrea & $10(11.1)$ \\
\hline Hemoptisis & $10(11.1)$ \\
\hline Reflujo gastro esofágico & $8(8.9)$ \\
\hline Sangrado digestivo & $8(8.9)$ \\
\hline Neurológicos & $5(5.6)$ \\
\hline Deshidratación & $5(5.6)$ \\
\hline Colelitiasis & $3(3.3)$ \\
\hline
\end{tabular}

\begin{tabular}{lc} 
Paraclínicos & \\
\hline Anemia $(11-16 \mathrm{~g} / \mathrm{dl}){ }^{* *}$ & $20(22.2)$ \\
Hipopotasemia & $6(6.7)$ \\
Hiponatremia & $4(4.4)$ \\
Hipoglicemia & $3(3.3)$ \\
Mortalidad & $22(24.1)$
\end{tabular}

Inmunohistoquímica

Sinaptofisina

Cromogranina

CD56

Sinaptogranina

* Media y la desviación estándar.

** Valor de referencia de hemoglobina institucional. n: tamaño muestral.
20. Sin embargo, en un estudio realizado en Colombia por Flórez y Col, la mediana fue de 48 años $^{[10]}$.

Los principales síntomas reportados fueron el dolor abdominal, pérdida de peso y tos. Lo anterior también se ha identificado en otros estudios en los cuales los dos primeros han sido los principales que han llevado al diagnóstico presentándose el primero hasta el $100 \%$ de los casos. El resto de los síntomas se presentaron en menor frecuencia con una gran diferencia con respecto a la literatura ${ }^{[11]}$

Los marcadores tumorales con mayor positividad fueron la cromogranina, sinaptofisina y CD56, similar a otros estudios ${ }^{21}$. Flórez y Col reportaron que los marcadores tumorales con mayor positividad fueron sinaptofisina $(97,2 \%)$, citoqueratinas AE1/AE3 (95\%), CD56 (91,3\%) y cromogranina $(87,8 \%)^{[12]}$.

En el presente estudio predominaron los tumores neuroendocrinos de intestino anterior, siendo el más frecuente el broncopulmonar, con gran diferencia, seguido de estómago y apéndice, lo cual difiere de lo reportado en la literatura. En el estudio de Yagmur y cols. en la distribución según el origen embriológico, fueron más frecuentes los de intestino medio ${ }^{[13]}$. De igual manera, en el $21,9 \%$ no fue posible establecer el origen primario del tumor, siendo una frecuencia alta comparada con la de otros estudios realizados y con la literatura ${ }^{[14]}$. En el estudio de Flórez y Col, fueron más frecuentes los tumores de origen pancreático $(34,8 \%)^{[15]}$, es decir en intestino anterior, diferente a nuestro estudio.

Teniendo en cuenta la última clasificación de tumores neuroendocrinos de la OMS del año 2019, que se basa en basa en los grados histológicos, en nuestro estudio, predominaron los carcinomas neuroendocrinos más de la mitad de los casos, contrario a otros trabajos realizados ${ }^{[16]}$. Sin embargo, dentro de los tumores neuroendocrinos bien diferenciados, las frecuencias de los diferentes grados histológicos solo tuvieron pequeñas diferencias, siendo más frecuente el de bajo grado o G1, similar a lo reportado en otras series ${ }^{[17]}$. El estudio PRONET reportó que la mayoría de los tumores, fueron tumores neuroendocrinos bien diferenciados, dentro de los cuales, más de la mitad son de bajo grado ${ }^{[18]}$. Otro estudio realizado teniendo en cuenta la última clasificación de OMS determinó que dos tercios fueron de bajo grado $^{23}$. En el estudio de Flórez y Col, predominaron los tumores bien diferenciados de grado intermedio o $\mathrm{G}^{[19]}$.

En este estudio, más de la mitad fueron tumores de alto grado, dentro de los cuales fueron más frecuentes los carcinomas neuroendocrinos, con una grande diferencia con respecto a los estudios mencionados. De igual manera en este estudio, fue más frecuente el carcinoma de células pequeñas, siendo diferente a lo reportado en otros estudios ${ }^{[20]}$. Las neoplasias de alto grado representan un bajo porcentaje, con pocos datos disponibles actualmente con respecto a las diferencias entre bien y mal diferenciados. Algunos estudios han reportado una frecuencia entre 14 y $18 \%$, de los cuales en un $20 \%$ fueron bien diferenciados. 


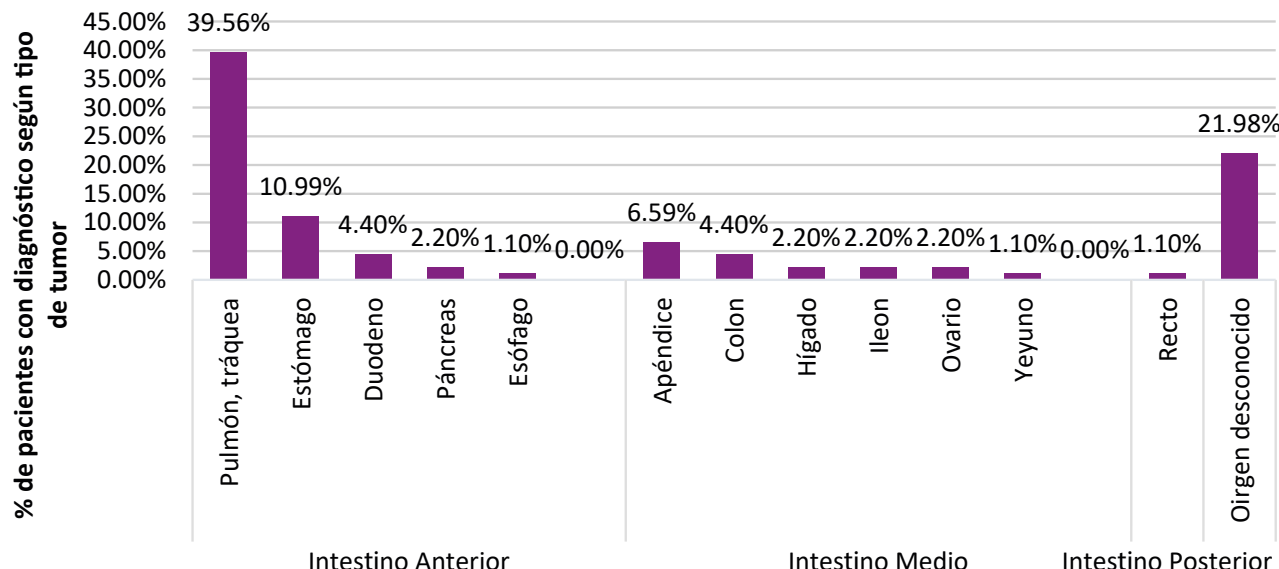

Tipo de tumor neuroendocrino por órgano afectado.

Figura 1: Origen de tumor por órgano afectado.

En el presente estudio, dentro de los tumores neuroendocrinos localizados en pulmón, una tercera parte fueron bien diferenciados, similar al reportado en otras investigaciones ${ }^{[21]}$, pero con grandes diferencias con respecto a los pobremente diferenciados, siendo menos frecuente en este estudio. De igual manera, se evidenció que hasta un tercio tenían antecedente de tabaquismo, menor comparado con el estudio realizado por Soto-Solís y col. ${ }^{[22]}$, reportaron que la mayoría de los tumores pulmonares fueron de alto grado ${ }^{[19]}$.

Las metástasis se reportaron en una tercera parte de los pacientes, principalmente hepáticas y ganglionar. Las primeras fueron comparables a otros estudios, pero con diferencias en las últimas ${ }^{[23]}$. En el estudio de Flórez y Col, también se reportó que las metástasis hepáticas fueron más frecuentes con un $73 \%$ de los casos ${ }^{[24]}$.

De los tumores neuroendocrinos localizados en pulmón, $29 \%$ fueron bien diferenciados y se observó el antecedente de tabaquismo datos comparable en la literatura: Se presenta alguna diferencia en presentación de los pobremente diferenciados que fueron $71 \%{ }^{[25]}$.

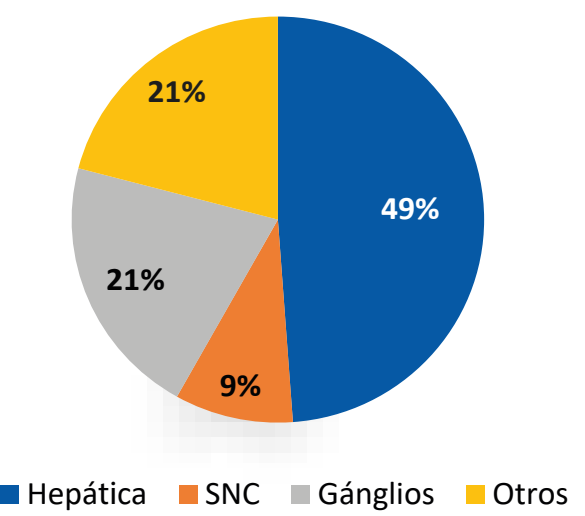

Figura 2. Órganos afectados por metástasis.
Los tumores neuroendocrinos rectales representan el $27 \%$ de los tumores neuroendocrinos gastrointestinales y el $16 \%$ de todos los neuroendocrinos, los gástricos el 9,6\%, los apendiculares 2-5\% ( 32 a $80 \%$ de los tumores apendiculares). Basado en los hallazgos, los tumores neuroendocrinos rectales solo representan el $1 \%$ de las neoplasias, lo que es diferente a lo reportado en la literatura, sin embargo, en origen gástrico y apendicular concuerdan con datos reportados. ${ }^{[26]}$ Aunque no se calculó mortalidad, durante el seguimiento de la corte se murieron el $24 \%$ datos que pueden similares en términos de mortalidad reportados en la literatura ${ }^{[27] .}$

Las diferencias en algunos de los resultados que presenta nuestro estudio pueden deberse a ubicación geográfica y la metodología utilizada entre los estudios con los cuales se ha comparado, sin embargo, no se descarta que estas diferencias puedan ser reales. De igual manera debe tenerse en cuenta que para el presente estudio se ha utilizado la nueva clasificación de la OMS 2019, lo cual dificulta la comparación en algunos datos, ya que la mayoría de los estudios han sido realizados con la clasificación 2010.

Entre las limitaciones están la ausencia de información en historias clínicas sobre tratamiento y tamaño tumoral que son susceptibles de pronostico y seguimiento, aunque no es la finalidad del estudio. El pequeño tamaño muestral no hace posible tomar conductas generalizadas, solo tomar la base de un registro de comportamiento poblacional específico de esta patología. Debido a las limitaciones del estudio con respecto a los pocos registros de algunos datos no es posible realizar curvas de supervivencia. Se requieren más estudios en nuestro país, que tengan un mayor nivel de evidencia y menos limitaciones, que nos permitan poder extrapolar los datos a otras regiones. No se realiza descripción de manejo quirúrgico, farmacológico o terapias adicionales por lo cual es paso a un estudio con mayor detalle que incluya estas variables.

El presente es un estudio con una cantidad importante de pacientes en una patología de baja prevalencia, con comportamiento demográfico y clasificación histológica y 
embriológica. El detalle de síntomas clínicos da bases a sospecha de tumores neuroendocrinos como experiencia de atención de esta patología.

Los tumores neuroendocrinos son una entidad de baja prevalencia y con comportamiento clínico variable. Las características clínicas e inmunohistoquímica del presente estudio no difieren significativamente de estudios reportados en la literatura mundial. Sin embargo, en cuanto al origen embrionario se observó mayor prevalencia de los tumores derivados del intestino anterior lo que difiere de reportes en los que derivan principalmente del intestino medio. A pesar de su diferencia esto no impactaría en el manejo de los pacientes. Los resultados expuestos pueden servir como estudio base para realizar investigaciones de mayor profundidad dada la gran importancia de este grupo de neoplasias en la población y por su baja prevalencia a nivel mundial.

Contribución de los autores: CM, DM, YG, MP, MV, SO, AP y HC concibieron y diseñaron la investigación, recolectaron los datos y, redactaron y aprobaron la versión final del artículo. CM diseñó e investigó el estudio base y preliminar del mismo. DM, YG, MV y $S O$ recolectaron la información, analizaron los datos y redactaron el artículo, AP Y HC Asesoraron la investigación.

Potenciales conflictos de intereses: Los autores del presente manuscrito declaran no tener ningún conflicto de interés financiero o no financiero, con relación a los temas descritos en el presente documento.

\section{Fuentes de financiamiento: Autofinanciado}

\section{ORCID}

Carlos Mauricio Martínez Montalvo: https://orcid.org/00000003-3136-4395

Deiner Yivelson Muñoz Delgado: https://orcid.org/0000-00016599-3830

Yeison Rodolfo Gómez Ledesma: https://orcid.org/0000-00026475-8633

Manuel Antonio Pérez Hettinga: https://orcid.org/0000-00032695-5211

María Daniela Vargas Calderón: https://orcid.org/0000-00030822-2786

Silvia Gabriela Ordoñez Muñoz: https://orcid.org/0000-00021462-1057

Alejandro Pinzón Tovar: https://orcid.org/0000-0002-6613-3602 Héctor Conrado Jiménez Sánchez: https://orcid.org/0000-00028856-1640

\section{REFERENCIAS BIBLIOGRÁFICAS}

1. González-Flores E, Serrano R, Sevilla I, Viúdez A, Barriuso J, Benavent $M$, et al. SEOM clinical guidelines for the diagnosis and treatment of gastroenteropancreatic and bronchial neuroendocrine neoplasms (NENs) (2018). Clin Transl Oncol [Internet]. 2019 Jan 7;21(1):55-63. Available from: $h$ ttp://link.springer.com/10.1007/s12094-018-1980-7
2. Guzmán $Y F$, López $R$ del P, Vera A, González-Devia D. Herramientas para el abordaje diagnóstico de los tumores neuroendocrinos de páncreas. Rev Colomb Cirugía [Internet]. 2018 Apr 27;33(1):79-99. Available from: https://www.revistacirugia.org/index.php/cirugia/ article/view/50

3. Liu D-J, Fu X-L, Liu W, Zheng L-Y, Zhang J-F, Huo Y-M, et al. Clinicopathological, treatment, and prognosis study of 43 gastric neuroendocrine carcinomas. World J Gastroenterol [Internet]. 2017 Jan 21;23(3):516-24. Available from: http://www.ncbi.nlm.nih.gov/ pubmed/28210088

4. Cidon EU. New therapeutic approaches to metastatic gastroenteropancreatic neuroendocrine tumors: A glimpse into the future. World J Gastrointest Oncol [Internet]. 2017 Jan 15;9(1):4-20. Available from: http://www.ncbi.nlm.nih.gov/ pubmed/28144395

5. Uppin MS, Uppin SG, Sunil CSPV, Hui M, Paul TR, Bheerappa $N$. Clinicopathologic study of neuroendocrine tumors of gastroenteropancreatic tract: a single institutional experience. J Gastrointest Oncol [Internet]. 2017 Feb;8(1):139-47. Available from: http://jgo.amegroups.com/article/view/12108/10140

6. Kaltsas G, Caplin M, Davies P, Ferone D, Garcia-Carbonero R, Grozinsky-Glasberg $S$, et al. ENETS Consensus Guidelines for the Standards of Care in Neuroendocrine Tumors: Pre- and Perioperative Therapy in Patients with Neuroendocrine Tumors. Neuroendocrinology [Internet]. 2017;105(3):245-54. Available from: http://www.ncbi.nlm.nih.gov/pubmed/28253514

7. Kulke $M H$, Benson AB 3rd, Bergsland E, Berlin JD, Blaszkowsky LS, Choti MA, et al. Tumores neuroendocrinos. J Natl Compr Canc Netw. 2012; 10 (6): 724-64.

8. Falconi M, Eriksson B, Kaltsas G, Bartsch DK, Capdevila J, Caplin $M$, et al. Actualización de las guías de consenso de la ENETS para el manejo de pacientes con tumores neuroendocrinos pancreáticos funcionales y tumores neuroendocrinos pancreáticos no funcionales. Neuroendocrinología. 2016; 103 (2): 153-71

9. Öberg K. La génesis del concepto de tumores neuroendocrinos: de Oberndorfer a 2018. Endocrinol Metab Clin North Am. 2018; 47 (3): 711-31.

10. Scarpa A, Chang DK, Nones K, Corbo V, Patch A-M, Bailey P, et al. Whole-genome landscape of pancreatic neuroendocrine tumours. Nature [Internet]. 2017;543(7643):65-71. Available from: http:// www.ncbi.nlm.nih.gov/pubmed/28199314

11 Coriat R, Walter T, Terris B, Couvelard A, Ruszniewski P. Gastroenteropancreatic Well- Differentiated Grade 3 Neuroendocrine Tumors: Review and Position Statement. Oncologist [Internet]. 2016;21(10):1191-9. Available from: http:// www.ncbi.nlm.nih.gov/pubmed/27401895

12 Zúñiga-Monge D. Tumores neuroendocrinos gastrointestinales. Med leg Costa Rica. 2013; 30(1):89-98. Available from: http:// www.scielo.sa.cr/scielo.php?script=sci_arttext\&amp;pid=\$140900152013000100012\&amp;Ing=en.

13 Scoazec J-Y, Couvelard A, Monges G, Guyétant S, Bisot-Locard $\mathrm{S}$, Parot $\mathrm{X}$, et al. Professional Practices and Diagnostic Issues in Neuroendocrine Tumour Pathology: Results of a Prospective One-Year Survey among French Pathologists (the PRONET Study). Neuroendocrinology [Internet]. 2017;105(1):67-76. Available from: http://www.ncbi.nlm.nih.gov/pubmed/27442514

14 Oronsky B, Ma PC, Morgensztern D, Carter CA. Nothing But NET: A Review of Neuroendocrine Tumors and Carcinomas. Neoplasia [Internet]. 2017 Dec;19(12):991-1002. Available from: http://www. ncbi.nlm.nih.gov/pubmed/29091800

15 Swart L, Schapkaitz E, Mahlangu JN. Thrombotic thrombocytopenic purpura: A 5-year tertiary care centre experience. J Clin Apher 
[Internet]. 2019 Feb;34(1):44-50. Available from: http://www.ncbi. nlm.nih.gov/pubmed/30536422

16 Moncet D, Flores M, Armanelli E, Carranza O, Zoppi J, Descalzo $\mathrm{J}$, et al. Tumores neuroendocrinos: Experiencia interdisciplinaria del hospital privado de comunidad TT -Neuroendocrine tumors: Interdisciplinary experience of the Private Community Hospital. Rev argent endocrinol metab [Internet]. 2019;56(1):30-9. Available from:http://www.scielo.org.ar/scielo.php?script=sci arttext\&amp;amp\%0Apid=S1851-30342019000100030

17 Flórez NF, Pérez Cadavid JC, Turizo Agámez Á, Cuesta Castro DP. Caracterización de pacientes con tumores neuroendocrinos en un hospital de referencia de alta complejidad. Rev Colomb Cirugía [Internet]. 2020 Oct 16;35(4):639-46. Available from: https://www. revistacirugia.org/index.php/cirugia/article/view/786

18 Varas-Lorenzo MJ, Cugat E, Capdevila J, Sánchez-Vizcaíno Mengual E. Detection of pancreatic neuroendocrine tumors: 23 years of experience. Rev Gastroenterol Mex [Internet]. 84(1):18-25. Available from: http://www.ncbi.nlm.nih.gov/pubmed/29858120

19 Soto-Solís R, Romano-Munive AF, Santana de Anda K, BarretoZuñiga R. Factors related to gastric neuroendocrine tumors. Rev Gastroenterol Mex [Internet]. 84(1):52-6. Available from: http:// www.ncbi.nlm.nih.gov/pubmed/29705524

20 Nagtegaal ID, Odze RD, Klimstra D, Paradis V, Rugge M, Schirmacher P, et al. The 2019 WHO classification of tumours of the digestive system. Histopathology [Internet]. 2020;76(2):182-8. Available from: http://www.ncbi.nlm.nih.gov/pubmed/31433515

21 Andreasi V, Partelli S, Muffatti F, Manzoni MF, Capurso G, Falconi $M$. Update on gastroenteropancreatic neuroendocrine tumors.
Dig Liver Dis [Internet]. 2021;53(2):171-82. Available from: http:// www.ncbi.nlm.nih.gov/pubmed/32912771

22 Alvarado-Cabrero I, de Anda-González J, Hernández-Hernández B, Mantilla-Morales A, Valencia-Cedillo R, MedranoGuzmán R. [Clinicopathologic characteristics of 127 cases of gastroenteropancreatic neuroendocrine tumors (GEP-NETs) studied in an oncology hospital]. Rev Gastroenterol Mex [Internet]. 77(4):174-80. Available from: http://www.ncbi.nlm.nih.gov/ pubmed/23142406

23 Medrano-Guzmán R. Guías de diagnóstico y tratamiento de tumores neuroendocrinos gastroenteropancreáticos. Patol Rev Latinoam. 2009;47(3):220-228.

24 Akın Telli T, Esin E, Yalçın Ş. Clinicopathologic Features of Gastroenteropancreatic Neuroendocrine Tumors: A Single-center Experience. Balkan Med J [Internet]. 2020;37(5):281-6. Available from: http://www.ncbi.nlm.nih.gov/pubmed/32573179

25 Yagmur Y, Söğutcü N, Gümüş S. Recent classifications systems for gastroenteropancreatic neuroendocrine tumors $\mathrm{A}$ single-center experience. Ann Ital Chir [Internet]. 2020;91:598-604. Available from: http://www.ncbi.nlm.nih.gov/pubmed/32989209

26 Heetfeld M, Chougnet CN, Olsen IH, Rinke A, Borbath I, Crespo $G$, et al. Characteristics and treatment of patients with $G 3$ gastroenteropancreatic neuroendocrine neoplasms. Endocr Relat Cancer [Internet]. 2015 Aug;22(4):657-64. Available from: http:// www.ncbi.nlm.nih.gov/pubmed/26113608

27 Flórez NF, Pérez Cadavid JC, Turizo Agámez Á, Cuesta Castro DP. Caracterización de pacientes con tumores neuroendocrinos en un hospital de referencia de alta complejidad. Rev Colomb Cirugía. 2020;35(4):639-46 